\title{
Macro EMG, a new recording technique
}

\author{
E STÅLBERG
}

From the Department of Clinical Neurophysiology, University Hospital, Uppsala, Sweden

SUMMARY A new electromyographic technique for the study of the motor unit is described. A modified single fibre EMG electrode is used. The electrical activity obtained by the electrode shaft during voluntary muscle contraction is averaged after triggering from a single muscle fibre action potential and the contribution from one motor unit is thus extracted. This "Macro EMG" signal gives information about the whole motor unit in contrast to the regional or focal electrical activity measured by conventional or single fibre EMG recordings, respectively.

The aim of an electromyographic investigation is to gain information about some of the properties of the motor unit and if possible about the individual muscle fibres constituting the motor unit. This is conventionally performed with various needle electrodes having different recording selectivities.

With the single fibre EMG (SFEMG) electrode having a $25 \mu \mathrm{m}$ recording surface, the uptake area is restricted to a hemisphere with a radius of about $300 \mu \mathrm{m}$ within which the action potentials have an amplitude of $0 \cdot 2-20 \mathrm{mV}$ and a duration less than $300 \mu \mathrm{sec}$. This uptake area includes 1 or 2 fibres when the motor unit is normal (fig. 1). The average number

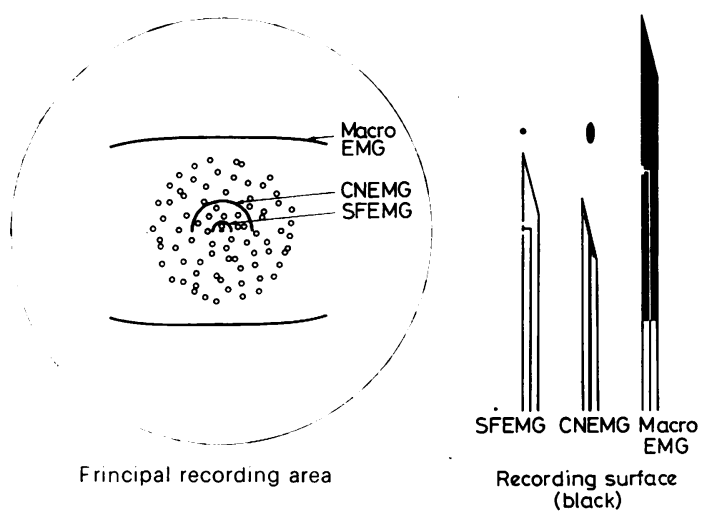

Fig 1 Focal, regional and global EMG. Recording area in the motor unit and active recording electrode surface for SFEMG, concentric needle EMG (CNEMG) and Macro EMG. In this study the whole cannula was active in the Macro EMG electrode, although a partly insulated electrode, as indicated here, will be recommerded.

Address for reprint requests: Dr E Stålberg, University Hospital, S750 14 Uppsala, Sweden.

Accepted 3 January 1979 of fibres belonging to one motor unit in 20 different sites is called the fibre density (FD) and differs slightly for different muscles and changes with age and with pathology. This recording technique is developed to be selective. It gives information about the individual muscle fibre (propagation velocity), its motor end-plate function (jitter) and about focal characteristics in the topography of the motor unit (FD). (For review see reference 1.)

The concentric needle electrode has a larger recording surface, $150 \times 580 \mu \mathrm{m}$ (fig 1). The spike component of the motor unit potential (MUP) recorded with this electrode is considered to reflect activity from muscle fibres within a radius of $1 \mathrm{~mm}$. Activity from more distant fibres is recorded with a similar amplitude from the active electrode surface and the electrode shaft, used as a reference, and is therefore cancelled to a great extent (fig 2). Since the normal motor unit in the biceps brachii muscle has an average diameter of 5 to $10 \mathrm{~mm}^{23}$ it is obvious that only a small proportion of all muscle fibres contributes to the main part of the so-called motor unit potential (MUP) recorded with a concentric needle electrode. Similarly with an average fibre density in the biceps brachii muscle of 1.35 fibres $/ 300 \mu \mathrm{m}$ radius this recording area covers about 15 muscle fibres. A similar value was obtained in an experimental study ${ }^{4}$ of the extensor digitorium communis muscle in which ischeamia was used to fractionate the motor unit; it was estimated that 5 to 12 muscle fibres contribute to the spike component of the MUP. Since the normal motor unit in biceps brachii has at least $\mathbf{2 0 0}$ muscle fibres ${ }^{5}$ the recording represents just a fraction of all its fibres.

With monopolar recording in which a subcutaneous electrode is used as reference the uptake area for muscle fibres generating the spike component is about the same as for a concentric needle electrode. Activity from distant fibres containing low fre- 


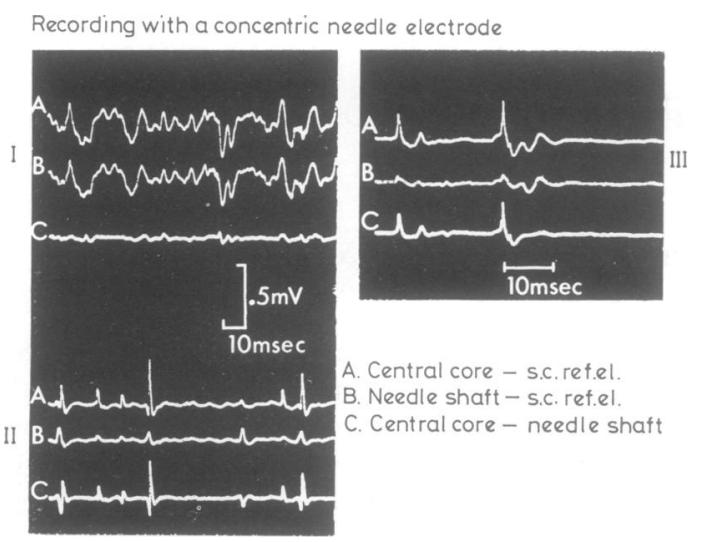

Fig 2 Motor unit activity recorded with the concentric needle electrode. In upper row $(A)$ derivation is made between the core (central platinum lead) and a remote subcutaneous reference electrode, corresponding to monopolar recording, in the second $(B)$ between the cannula and the subcutaneous reference and in the third $(C)$ between the core and the cannula (the ordinary recording). In I the electrode tip is outside the muscle. The same activity is recorded from core and cannula. In II and III the electrode is in the muscle. The cannula usually records some activity from the same motor unit as the core but with another shape. In III it is seen that the conventional derivation reduces the distant contribution to the MUP, which therefore gets a shorter duration. In II some action potentials are recorded from the cannula but not from the core. In the ordinary derivation this is indicated by reversed polarity.

quencies also contributes to the recorded signal and is not cancelled as in concentric needle EMG recording. The MUP therefore has somewhat different characteristics than when obtained with the concentric needle electrode.

More general information about the motor unit may be obtained with surface electrode recordings where all fibres belonging to one motor unit contribute to the recorded signal more equally, since the relative distance to the electrode for the individual fibres differs less than with more selective recor dings. The contribution to the surface EMG from one individual motor unit can be obtained in the following way. Recordings are made with a pair of surface electrode silver strips or discs, one over the belly of the muscle and one over a silent area (antagonistic side). The signal obtained during voluntary muscle contraction is fed to an averager triggered by a intramuscularly inserted single fibre EMG electrode recording selectively from one of the muscle fibres of the motor unit under study. With this technique potentials are obtained with amplitudes varying between 25 and $80 \mu \mathrm{V}$ for the biceps brachii muscle ${ }^{6}$ These voluntarily activated motor unit potentials correspond to those obtained with the modified motor unit counting technique of Ballantyne and

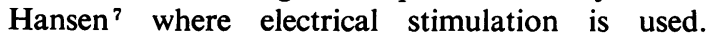
However, the distance from the centre of the different motor units to the surface varies and the amplitude is therefore to a significant degree dependent on the location of the motor unit within the muscle. Maintaining the principal of making the recording with a relatively large electrode but in a better defined position relative to the active motor unit than that obtained with surface electrodes, a special electrode was constructed (fig 1) to record the "Macro EMG." In this study, the method is discussed and some results from recordings with such an electrode are presented.

\section{Method}

The electrode consists of a $50 \mathrm{~mm}$ steel cannula with a side port $10 \mathrm{~mm}$ behind the tip exposing a $25 \mu \mathrm{m}$ diameter platinum wire. Recording is made on one channel from the small electrode with the cannula as reference (ordinary single fibre EMG recording). On the other channel the derivation is made between the cannula and a remote subcutaneous concentric needle electrode as reference (fig 2), placed at least $30 \mathrm{~cm}$ away. The filter settings have been $500 \mathrm{~Hz}$ to 8 $\mathrm{kHz}$ for the single fibre EMG channel and $8 \mathrm{~Hz}$ to $8 \mathrm{kHz}$ for the Macro EMG. Values lower than $8 \mathrm{~Hz}$ can be used for the Macro recording but the recording will then show marked DC shifts of the
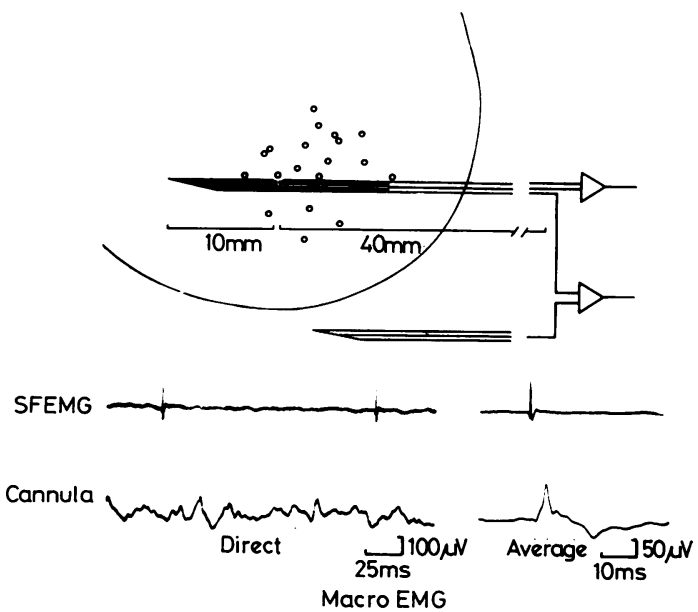

Fig 3 Recording principle. The small surface in the Macro EMG electrode is positioned to record action potentials from one muscle fibre. This is used to trigger the averager to which activity recorded with the cannula is fed. After 128-512 discharges the synchronous motor unit activity is extracted. In this study the whole cannula was active in the Macro EMG electrode. 
baseline with electrode movements. Values higher than $8 \mathrm{~Hz}$ will distort the results since the signal contains components from this frequency range. A practical test does not show any difference between recordings from the same motor unit when $8 \mathrm{~Hz}$ and $1.6 \mathrm{~Hz}$ have been used.

The original signals or averaged potentials can be stored undistorted by an FM tape recorder (DC to $2500 \mathrm{~Hz}$ ) but an AM tape recorder cannot be used because of non-linearity in the lower frequency response curve and because of insufficient low frequency response below $20 \mathrm{~Hz}$. Our recordings are analysed on line.

The electrode is inserted into the silghtly voluntarily activated muscle and positioned to record a single fibre action potential with high amplitude from the small electrode. The patient is asked to maintain a minimal but constant activation with the action potential from the small recording surface firing at a regular rate, usually about 10 impulses per second. This action potential is used to trigger a digital averager to which the signal from the large recording surface, the cannula, is fed and averaged, usually for 128 to 512 impulses. The signal is delayed by $40 \mathrm{~ms}$ after the trigger to allow detection of early components.

The analysis of the obtained signal has been made by means of a computer (PDP 11/40 or LSI-11) on line. Peak to peak amplitude is measured. Due to the shape of the response and background noise, it is usually difficult to define a duration measure. The area has been calculated in the following way. A "signal window" of $60 \mathrm{~ms}$ is established, with its midpoint at the triggering potential. A baseline is determined by averaging the activity $10 \mathrm{~ms}$ prior to and $10 \mathrm{~ms}$ after the $60 \mathrm{~ms}$ period, and connecting these two averaged values by a straight line. The area under the envelope of the signal is calculated.

The shape of the Macro EMG response is determined by the temporal and spatial summation of the individual single fibre potentials within the motor unit. The optimal position for the recording electrode is perpendicular to the main fibre direction. Any deviation from this will theoretically introduce errors in the motor unit parameters, usually towards a lower amplitude and somewhat prolonged duration but the opposite is also possible in some situations, eg when the motor end-plate zone is skewed. The correct angular position is more important with this electrode than with conventional EMG or single fibre EMG electrodes. Rotation of the electrode does not change the responses as it does in conventional and particularly in single fibre EMG recordings. In the biceps brachii and the tibialis anterior muscles used in the present study the main fibre direction is easy to determine and the electrode position error is probably insignificant. When other muscles are investigated with more complicated fibre directions a standardised needle position must be used to minimise the effect of position error.

The relation to the end-plate zone may influence the recording since the temporal dispersion increases towards the tendon. The magintude of this effect will be further investigated. In the present study the electrode has been positioned in the middle third of the muscles.

\section{Results}

The biceps brachii and tibialıs anterior muscles have been investigated in seven healthy subjects aged 15 to 36 years. 190 and 164 motor units respectively have
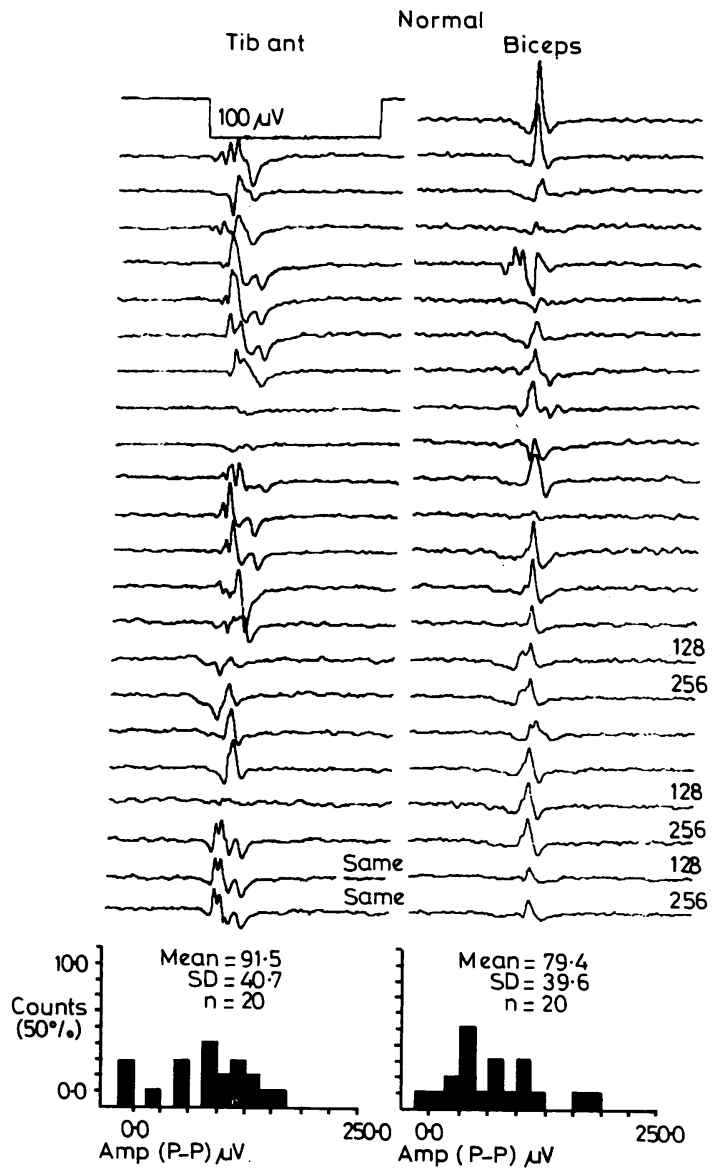

Fig 4 Macro EMG recordings from 2$)$ different recording sites in biceps brachii and tibialis anterior muscles respectively in a healthy subject. Some of the recordings are shown twice with a different number of discharges for averaging. Amplitude histograms of the depicted potentials are shown at the bottom. 

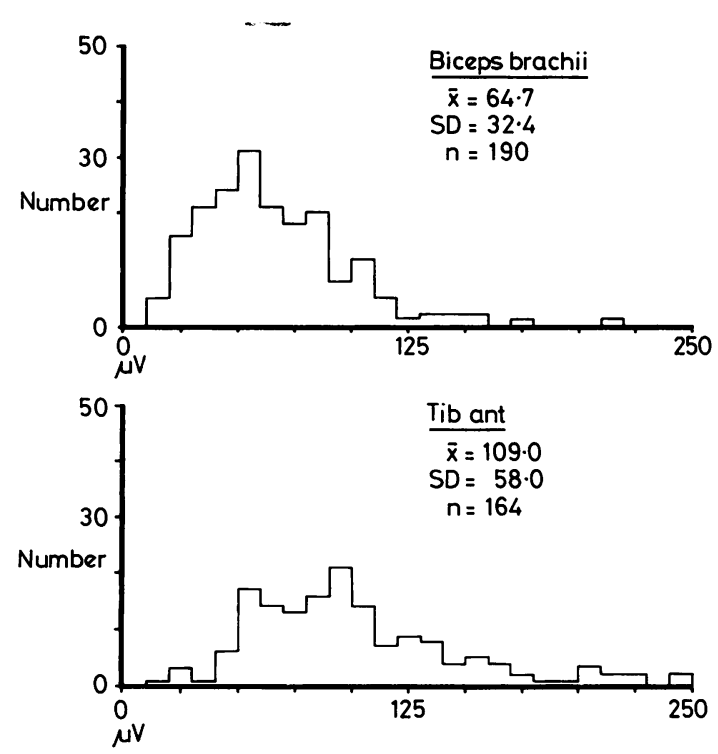

Fig 5 Amplitude distribution of Macro EMG potentials from biceps brachii and tibialis anterior muscles from 7 healthy subjects.

been studied. The peak to peak amplitude was $64 \cdot 7 \pm 32 \cdot 4 \mu \mathrm{V}$ (range 11 to $218 \mu \mathrm{V}$ for individual motor unit potentials) and $109 \pm 58.0 \mu \mathrm{V}$ (range 18$291 \mu \mathrm{V})$ for biceps brachii and tibialis anterior muscle (figs 4,5 ), respectively. The mean value differed as much as $100 \%$ between different healthy subjects, with the lowest values being found among

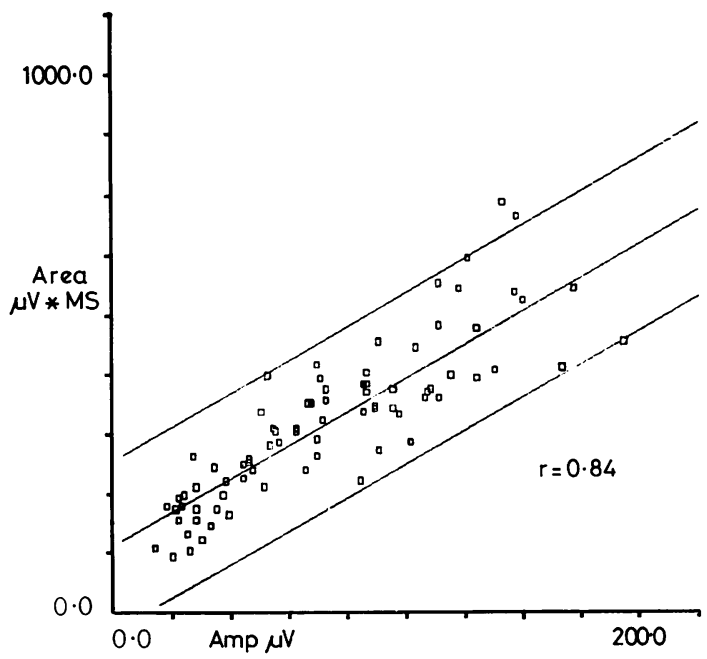

Fig 6 Correlation between amplitude and area of 100 Macro EMG potentials from normal muscles. There is a positive and significant correlation between these two parameters.

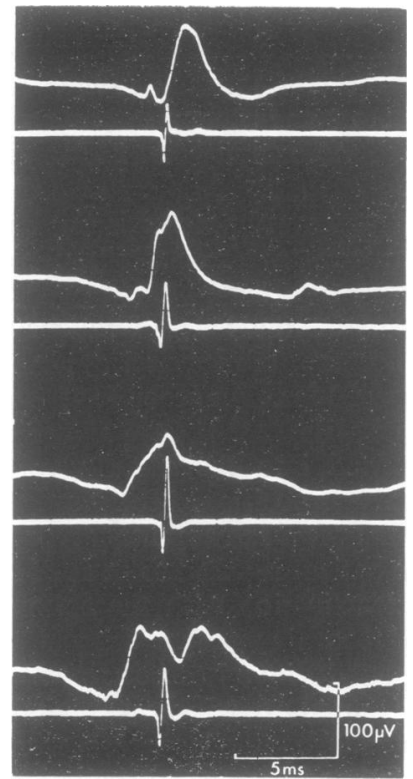

Fig 7 Examples of actual recordings showing the average Macro EMG (first line) and the triggering action potential (second line). The triggering action potential does not necessarily occur in the earliest part of the motor unit potential. Note that the contribution to the Macro EMG from the triggering action potential is usually not seen. Recording from tibialis anterior muscles in a healthy subject.

the youngest subjects in the group. The area was $273 \pm 108 \mu \mathrm{V} \times \mathrm{ms}$ and $364 \pm 134 \mu \mathrm{V} \times \mathrm{ms}$ for the two muscles. There was a positive and significant correlation $(r=0.84)$ between the area and the the amplitude (fig 6).

The arrival time to the electrode for the triggering action potential corresponded grossly to the obtained MUP but could occur with any latency within the response (fig 7). The triggering potential could sometimes be seen in the MUP as a notch, less than $5 \mu \mathrm{V}$. Usually it was not seen at all. In case of fibrillation potentials no Macro response was obtained. There was no correlation between amplitude or area and fibre density in the normal muscle.

In order to study the shape of the MUP in different electrode positions within the same motor unit the following procedure was used. A separate single fibre EMG electrode was used to record single fibre action potentials from one motor unit and to trigger the oscilloscope. The Macro EMG electrode was inserted and a position was sought where the small electrode surface recorded an action potential synchronous to the triggering potential from the single fibre EMG electrode, that is appearing at a constant position on the oscilloscope screen, indicating that the two electrodes were recording activity from the same motor unit. Now the Macro EMG from the cannula was recorded. With the separate triggering electrode in a constant postition this procedure was repeated 2 to 7 times in the same motor unit with different skin insertions of the Macro EMG electrode, each spaced about $5 \mathrm{~mm}$ from the previous insertion along the muscle. Some examples 


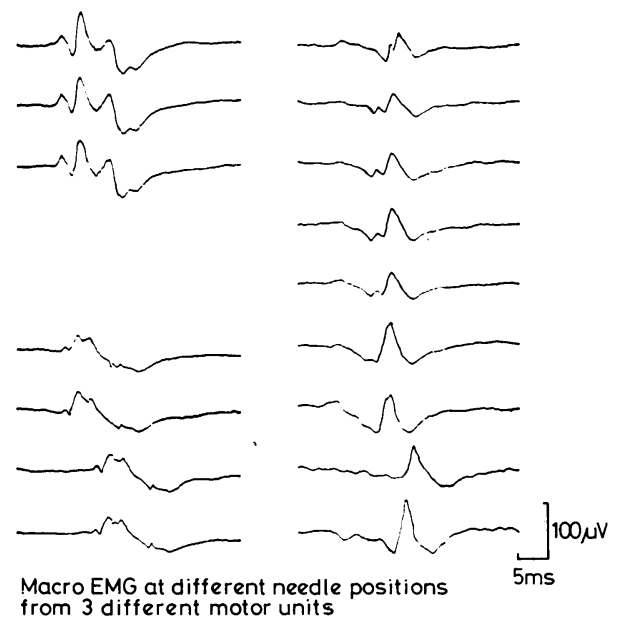

Fig 8 Macro EMG recording from 3 different motor units. Within each motor unit the recording sites $(3,4$ and 7 respectively) are separated by at least $5 \mathrm{~mm}$ along the muscle towards the tendon. Note the relative constancy of the potential shapes of each motor unit recorded at the different sites.

are shown in fig 8. It is seen that the shape of the recorded potential varies more between different motor units than within the same motor unit. The constancy in Macro EMG can also be seen in a special scanning recording described elsewhere ${ }^{8}$.

\section{Comparison to surface recordings}

A comparison was made between the motor unit potential recorded with surface electrodes, one silver strip as recording electrode over the belly of the biceps brachii muscle and one as reference placed below the elbow, and that recorded with the cannula. The action potentials recorded from the small surface in the needle electrode served as trigger for the Macro EMG and the surface recordings which were made simultaneously. A two channel recording system (Medelec MS6) with signal delay line (SDS6) and averager (DAV62) was used. The Macro EMG needle recordings were specifically made either relatively superficially in the muscle or deep. The results are summarised in the table. In general the amplitude and area were lower for the surface EMG recordings. The relative difference was larger for the deep motor units. Overall, there was a correlation for amplitude and area between needle and surface recordings. This correlation was statistically significant for the superficial motor units and for area but not amplitude for the deep motor units.
Table Comparison between Macro EMG and surface EMG responses when recording superficially and deep in normal biceps brachii muscle. Surface EMG response is generally lower, particularly when deep motor units are recorded. There is correlation between the Macro and surface EMG responses except for the amplitude parameter in deep recordings.

\begin{tabular}{|c|c|c|c|c|}
\hline & \multicolumn{2}{|c|}{ Superficial motor units } & \multicolumn{2}{|c|}{ Deep motor units } \\
\hline & $A m p l \mu V$ & Area $\mu V \times m s$ & $A m p l \mu V$ & Area $\mu V \times m s$ \\
\hline $\begin{array}{l}\text { Macro EMG (M) } \\
(n=20)\end{array}$ & $72 \pm 37$ & $311 \cdot 7 \pm 125 \cdot 5$ & $50 \pm 28$ & $194 \cdot 8 \pm 69 \cdot 3$ \\
\hline $\begin{array}{l}\text { Surface EMG (S) } \\
\quad n=20)\end{array}$ & $32 \pm 18$ & $216 \cdot 5 \pm 142 \cdot 9$ & $12 \pm 6$ & $95 \cdot 2 \pm 51 \cdot 9$ \\
\hline $\begin{array}{l}\text { Corr } \mathbf{M} \text { vs } \mathbf{S} \\
\text { Quotient } \mathbf{M} / \mathbf{S}\end{array}$ & $\begin{array}{c}0.62 * \\
2 \cdot 3\end{array}$ & $\begin{array}{l}0.57^{*} \\
1.4\end{array}$ & $\begin{array}{l}0 \cdot 3 \mathrm{NS} \\
4 \cdot 2\end{array}$ & $\begin{array}{l}0 \cdot 53^{*} \\
2 \cdot 0\end{array}$ \\
\hline
\end{tabular}

$*=p<0.05$

Reference electrode as a source of error Since the activity from one motor unit, particularly when superficially located, can be recorded with considerable amplitude on the surface of the muscle it is important to ascertain that the reference electrode used for Macro EMG does not record any activity from the muscle under study. To achieve this, the reference can be made as selective as is practical, for example by using the leading-off surface of a concentric needle electrode. In addition the reference must be placed in an area remote from the muscle under study. With increasing distance between recording and reference electrode the risk of external interference increases however and an optimal distance should be used. To study the amount of "contamination" to the reference electrode the motor unit was triggered with an intramuscular electrode in the biceps brachii muscle and recording was made between the leading-off surface of a subcutaneous concentric needle electrode (later to be used as reference in macro recordings) at different positions against a reference electrode on the other arm. It was found that the motor unit potential from the biceps brachii muscle could often be seen even when the electrode was placed over the tendon regions of the muscle but not when it was placed over the deltoid muscle or below the elbow. Therefore we have chosen to place the electrode over the head of radius when recording from the biceps brachii and lateral to the patella when recording from the tibialis anterior muscle.

Tremor or unsteady firing as a source of error The averaging technique used to extract the activity from one motor unit by triggering on the action potential from one of its fibres is based on the assumption that the activity from other motor units occur completely randomly in relation to the one under study. This is true at slight and moderate contractions giving con- 
stant force. If tremor occurs, however, activity from other motor units is likely to occur nearly synchronously with the triggerıng unit and will therefore systematically contribute to the averaged response. Tremor or unsteady firing is more common in recordings from the normal tibialis anterior than from the biceps brachii muscle and may also ozcur in fatigued muscle and in some pathological conditions. The importance of this effect was studied by inducing jerky movements voluntarily. The amplitude of the recorded response could increase by $100 \%$ (usually less than $50 \%$ ) and the area by four times in extreme cases. This is a technical problem which may lead to overestimation of the response size. It is most important when the triggering motor unit does not have the lowest threshold among those in the recording area but appears with some delay after the initiation of the tremor burst. During recording from a very low threshold motor unit the tremor does not disturb the results very much, at least not the amplitude parameter since the "contamination" is seen in the later part of the recorded response. Therefore the original "cannula signal" should always be monitored at least by the loud speaker and if possible as an integrated EMG signal. Indications of tremor should disqualify the recording. Only a tremor-free recording can be accepted.

Pathological motor units. In a few patients with neuropathies, we compared fibre density, concentric needle EMG and Macro EMG. The Macro EMG action potential is usually increased in neurogenic disorders (fig 9). There was a general agreement between the results but some discrepancies were

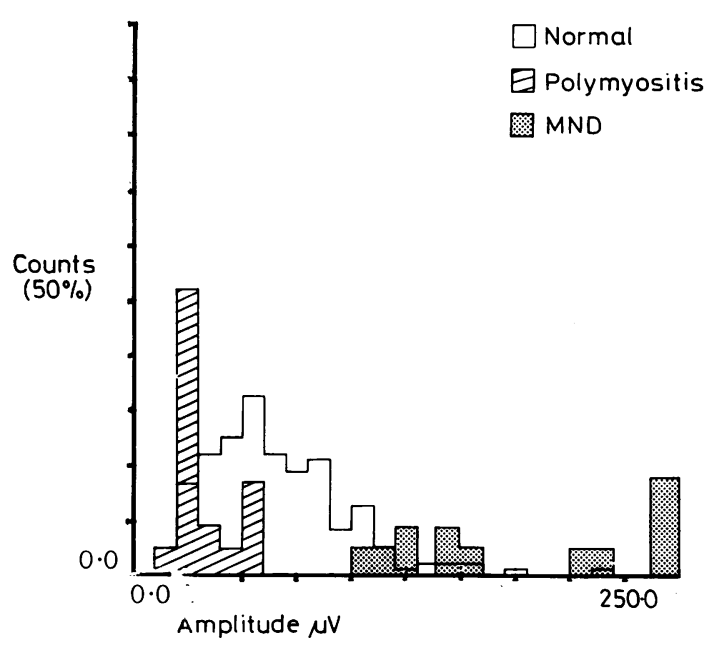

Fig 9 Macro EMG findings in a case of polymyositis and motor neuron disease (MND) from biceps brachii muscle compared to normal.

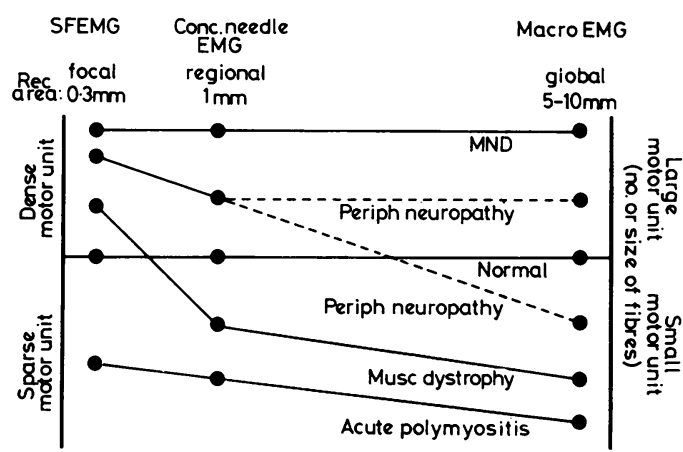

Fig 10 A schematic comparison between SFEMG, concentric needle EMG, and Macro EMG.

obtained. In one patient receiving chloroquine for rheumatoid arthritis, who had reduced motor nerve conduction velocities, the fibre density was clearly increased and the concentric EMG showed increased amplitude and long duration potentials with a reduced recruitment pattern in biceps brachii and tibialis anterior muscles. The Macro EMG amplitude was increased in the biceps brachii muscle but decreased to $20 \%$ of the normal in tibialis anterior muscle. The biopsy showed marked muscle changes consisting of fibre type grouping and muscle destruction with fibrosis particularly in the tibialis anterior muscle.

In myopathies the Macro EMG action potential amplitude is normal or decreased (fig 9) parallel to the concentric EMG while the fibre density is increased. A sc'lematic summary is given in fig 10 .

\section{Discussion}

The single fibre EMG electrode was developed to allow more selective recording than can be obtained with concentric needle electrodes. This is accomplished by using a small recording surface from which the action potential from a very close muscle fibre is recorded with minimal distortion and therefore with high amplitude. The larger electrode surface in the concentric electrode causes a shunting effect and the amplitude of the closest fibres is "erroneously" low. Action potentials from distant fibres are recorded with about the same amplitude by the two electrodes. Thus, the difference in amplitude of action potentials from adjacent and distant fibres is more pronounced with the small electrode, giving higher recording selectivity than the larger electrode.

With a still larger electrode such as the steel cannula used in Macro EMG this shunting effect is more pronounced and the difference in contribution to the recorded signal between adjacent and distant fibres is even smaller than in the concentric electrode. 
Furthermore, because of the physical size of the large electrode (the cannula) the whole normal motor unit is penetrated and therefore a larger number of muscle fibres in the motor unit are closer to some part of the recording surface. The triggering electrode is located $10 \mathrm{~mm}$ behind the tip of the cannula (more than the average normal motor unit diameter) so that if the triggering fibre is at the margin of the motor unit the entire cannula has still pierced the whole territory. The recorded signal is therefore theoretically a better measure of the electrical power of the total motor unit than is the action potential obtained with concentric needle EMG which has an uptake radius of about $1 \mathrm{~mm}$ for the main part of the MUP.

The relative nonselectivity of the Macro EMG, that is its reflection of the total motor unit signal, is demonstrated in the studies where the same motor unit was recorded at quite separate locations along the muscle. The main characteristics of the action potential remained although a continuous change for different sites could be detected. Due to the different propagation velocity for the different fibres this change in shape of the action potential is expected.

The shape of the signal is dependent on the spatial distribution of the muscle fibres with low amplitude from distant fibres. Also the temporal distribution of the individual action potentials determines the shape of the recorded signal. From other studies ${ }^{8}$, it is obvious that the temporal distribution in a transverse cross-section of the motor unit varies considerably for different motor units even in the normal muscle. Assuming a correct needle position, that is perpendicular to the fibre direction, the temporal dispersion is dependent on the conduction time along the nerve branches, the anatomic location of the motor end-plates and the propagation velocity in individual muscle fibres. These factors will sometimes cause double or multiple peaks in the recorded response (fig 5). The total amplitude is therefore not an ideal measure to quantify the response. The duration has not been used yet as a parameter, since difficulties arise in defining the start and the stop of the potential, particularly in pathological situations. We have therefore chosen to use the area under the potential as one measure. Since the peak-to-peak amplitude both in normal and pathological conditions generally shows a good correlation to the area, and is easy to measure without computer, it is at present used as well as the area as a parameter of the motor unit porential size.

The single fibre EMG represents one end of the scale of electrophysiological methods of studying the motor unit signal, the Macro EMG the other and the concentric EMG is somewhere between. Single fibre
EMG gives information about local fibre density within a $0.3 \mathrm{~mm}$ radius around the active electrode, the concentric EMG represents the motor unit within $1 \mathrm{~mm}$ and the Macro EMG represents the major part of the motor unit. The Macro EMG signal can therefore in contrast to conventional EMG be used as a measure of the motor unit size including the number of fibres and their individual size. This may be of interest in many neurophysiological situations both in routine clinical studies and in research.

What seem to be discrepant results between single fibre EMG and concentric EMG on the one hand and Macro EMG on the other may point to the potential value of the new technique. The increased fibre density in muscular dystrophies combined with low action potentials in concentric EMG and Macro EMG is interpreted as a local densification of fibres due to splitting, regeneration or reinnervation within $0.3 \mathrm{~mm}$ of the active electrode but reduced number of fibres of the motor unit within $1 \mathrm{~mm}$. The findings in the reported case of neuropathy with increased fibre density and high amplitude signal in the concentric EMG together with reduced Macro EMG action potential can be interpreted as a densification within $1 \mathrm{~mm}$ but a reduced total size of the motor unit. This interpretation was supported by biopsy findings.

In the single fibre EMG and concentric needle EMG recordings, "silent areas" within the motor unit (larger than 0.3 and $1 \mathrm{~mm}$ in radius respectively) are not included in the overall results as they are in Macro EMG. Particularly in pathological conditions with uneven fibre distribution within the motor unit these methods therefore do not give a true picture of the average activity of the motor unit.

The Macro EMG method can relatively easily be adopted in an EMG laboratory. A special needle electrode, two amplifiers, trigger, signal delay and averaging facilities are necessary. It usually takes less than one minute to study one motor unit. For the patient the investigation is similar to conventional EMG.

Further experience with the Macro EMG technique in normal muscles and in different neuromuscular disorders will hopefully show whether and where it can be used as a valuable additional method for the study of the motor unit.

After the completion of this study the electrode has been slightly modified. The cannula is now insulated but for a distance of $15-20 \mathrm{~mm}$ from the tip which is used for recording. This is already indicated in figs 1 and 3. The main reason for this is to reduce the variation in shunting effect on the recorded signals depending on length of metal shaft in the muscle. The lower amplitudes of the recordings 
from the deep motor units (table) is partly due to this phenomenon. The amplitudes of the responses recorded with the partly insulated needle are on average more than twice those obtained with the non-insulated cannula. Normal values are collected with this electrode.

The investigation was supported by the Swedish Medical Research Council (Grant No. 135). I express my gratitude to eng. G. Lovén who made the electrode, to eng. L. Antoni who wrote the computer programs, and to Dr D B Sanders who reviewed the manuscript.

\section{References}

1 Stålberg, E and Trontelj, J. Single Fibre Electromyography. Woking, England: Mirvalle Press, 1979.

2 Buchthal, F, Guld, Ch and Rosenfalck, P. Multielectrode study of the territory of a motor unit. Acta Physiol. Scand. 1957; 39:83-103.
3 Stålberg, E, Schwartz, MS, Thiele, B and Schilleir HH. The normal motor unit in man. J Neurol Sci 1976; 27:291-301.

4 Thiele, B and Boehle, A. Number of single muscle fibre action potentials contributing to the motor unit potential. 5th Internat Congr of EMG. Rochester, Minn, 1975.

5 Stålberg, E, Gath I. In situ measurements of the innervation ratio of motor units in human muscles. In press, IEEE Transactions on Biomedical Engineering.

6 Stålberg, E. Electrogenesis in human dystrophic muscle. In: Rowland LP, ed. Pathogenesis of Human Muscular Dystrophies. Proc of the 5th Internat Congr of the Muscular Dystrophy Assoc. Excerp Med 1977; 570-87.

7 Ballantyne, JP, Hansen, S. A new method for the estimation of the number of motor units in a muscle. Control subjects and patients with myastehnia gravis. $J$ Neurol Neurosurg Psychiat 1974; 37:907-15.

8 Stålberg, E, Antoni, L. The electrophysiological cross section of the motor unit. $J$ Neurol Neurosurg Psychiatry 1980; 43: 469-74. 